antipathy to the use of radioactive substances, this factor may prove a social rather than biomedical stimulus.

The drawbacks of nuclear magnetic resonance should not, however, be played down. Data acquisition is slow, of the order of 10 minutes; the equipment is costly-of the same order as a computed tomography scanner-and bulky: a whole-body nuclear magnetic resonance unit two metres long and one metre in diameter weighs about $4000 \mathrm{~kg}$ (4 tons) and contains superconducting magnets with their problems of handling liquid nitrogen and helium.

Since units are now available commercially, studies to establish the clinical utility of nuclear magnetic resonance must be of high priority. As yet we do not know whether it will be an adjunct to computed tomography or whether it will supplant it. Since up-to-date installations of either kind cost about $£ 500000$ we cannot realistically conceive of them operating side by side in the average district general hospital.

\section{Emission computed tomography}

Emission radiography, using injected or inhaled radionuclides, is not a new technique but it has developed recently as a result of the application of data acquisition and, more importantly, data analysis technology. Indeed, work in the latter antedated $x$-ray computed tomography.

While there is no doubt that the diagnostic accuracy of isotope studies has thereby been increased, application of the results has been limited by poor spatial resolution, lack of specificity, and an inability to display, for example, some cerebral structural lesions without blood-brain barrier defects. Even with the most refined techniques, emission tomography seems most promising for metabolic studies. With the use of short-lived isotopes such as oxygen-15 or deoxyglucose labelled with fluorine-18, transverse sectional images of the brain can be constructed to give quantitative information about regional blood flow, oxygen extraction and utilisation, or glucose uptake. Extremely interesting data on the balance between perfusion and tissue necrosis in ischaemic infarction have already been obtained that could in the long term persuade us to modify our views on the management of stroke (fig 5).

Emission tomography is essentially non-invasive and relatively inexpensive, but its diffusion will be restricted by the need for access to a cyclotron for the short-lived isotopes.

\section{Conclusions}

Radiology made relatively few fundamental advances in its first 75 years as a specialty, while in the past 10 years quite unforeseen changes have occurred. At the recent Radiological Societies of North America Exhibition in Chicago (the Motor Show of the radiology industry) many of the major manufacturers unveiled their new computed tomography scanners, digital angiography units, and nuclear magnetic resonance imagers. In my own hospital the annual medical equipment budget for all departments combined would fall short of the price of any one of these. While good clinical medicine must

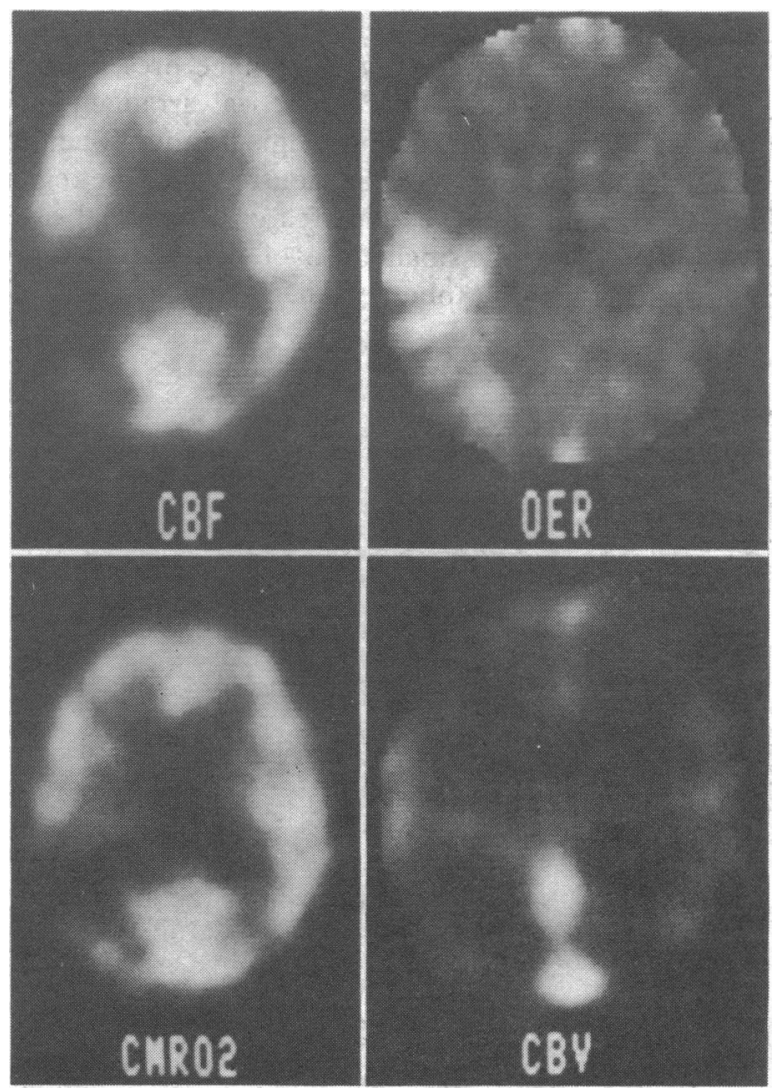

FIG 5-Positron emission CT. Scan performed eight hours after cerebral infarct. There is focal reduction (black area) in cerebral blood flow (CBF), reflected by avid cygen extraction (OER), which is insufficient to maintain oxygen consumption (CMRO2). This pattern of "critical perfusion" thought to indicate acute ischaemia. pattern of "critical perfusion" thought to indicate acute ischaemia. Physics Isotope Section, MRC Cyclotron Unit, Hammersmith Hospital, London.)

come first, we must not forget that most of these recent developments aim to extend our diagnostic range and to replace erstwhile invasive procedures with simple, reliable, anodyne, and cost-effective screening methods. To rule out widespread use of modern technology because of the major financial investment needed could prove shortsighted.

IVAN MOSELEY

Consultant Radiologist,

National Hospital,

London WCIN 3BG

\section{Correction}

\section{Schools of public health}

The personal review by Professor Roy Acheson (20 February, p 534) wrongly stated that Professor Le Quesne had recommended that the department of nutrition should move from the London School of Hygiene and Tropical Medicine. Professor Le Quesne in fact said: "The distinguished work of this department might strengthen its links with clinical medicine." 